Newborn Screening: Now and in the Future

Rachel Donnison

Editorial Administrator

Citation: EMJ. 2020;5[2]:12-13. Congress Review.

$\star W$

AT is the value of newborn screening (NBS) to the rare diseases community? This was the question posed to the audience by Nick Meade, of Genetic Alliance
UK, at the session 'Newborn Screening: Now and in the Future' at the European Congress of Rare Diseases and Orphan Products on 14th May 2020. Given the wide range of credentials in the audience, with patient representative organisations making up 33\% of those in attendance, followed by academics and industry representatives both at $14 \%$ and healthcare professionals at 11\%, it is clear that the value of NBS is acknowledged throughout the rare disease community.

\section{INTERNATIONAL VARIATION IN}

\section{PRACTICE}

Country-to-country changes in NBS are common as highlighted by Prof Martina Cornel, Chair of the Netherlands Programme Committee on Neonatal Screening. Neonatal screening programmes were introduced in the 1960s after the finding that phenylketonuria, affecting around 1 in 18,000 newborns, was treatable if diagnosed early. Stressing that "a programme is more than a test," Cornel highlighted the patient information, communication, and follow-up needed. The landscape of neonatal screening in Europe in 2010 was, and to some extent continues to be, widely varied: only 18 of the 35 countries surveyed had a NBS steering committee and, of those that did have a screening programme, the number of diseases included in the screening ranged from 25 in Portugal to $\mathrm{O}$ in Albania. Since 2010, several initiatives have been created to harmonise NBS across Europe, as Cornel underlined that "whether a child was born in one country or another country made a big difference in terms of earlier recognition of rare diseases." In the future, Corne recommends attuning the three perspectives related to NBS: public health, commercial and industry, and parents and patients.

\section{EXPANDED NEWBORN SCREENING IN}

\section{ITALY}

Not all nations take the same approach, and Simona Bellagambi, elected member of the Board of Directors of Rare Diseases Europe (EURORDIS), detailed the level of expansion in taly's NBS initiative. The country has come a long way since the introduction of screening in 1992 which included screening for just three conditions (phenylketonuria, congenital hypothyroidism, and cystic fibrosis). Fast forward to 2016 and an Italian ministerial decree increased the panel to include 40 screened diseases. By 2019, new amendments included other conditions such as lysosomal diseases and primary immunodeficiencies and a compulsory 2-year update of the panel.
Additionally, in 2020 and 2021 there will be annual increases of $€ 2$ million in country-wide funding, to bring the total to $€ 33,715,000$ for the inclusion of new pathologies into the programme. However, Bellagambi still believes more can be done: "we are very much aware that we are still missing a working group for the elaboration of the operative protocol." A complex and multidisciplinary system, Bellagambi organised meetings with patient organisations to raise awareness of the expanding NBS programmes in Italy and collated all patient needs pre- and post-testing. This resulted in a position paper with 10 recommendations, which Bellagambi says "addresses the issues of the protection of the human rights in the processes of carrying out expanded newborn screening." Emphasising the importance of equality, with full implementation in all regions; extension, so that screening is available for all pathologies with a treatment; and utilising biobanking to further use the residual material of bloodspots for research purposes, Bellagambi does not stop there, and advocates for future inclusion of neonatal screening of all genetically transmitted diseases, even if currently incurable, as expanded NBS saves lives.

\section{A CASE STUDY OF}

Providing a patient perspective, the next speaker was Sarah Hunt, CEO of Alex TLC, a charity she was Sarah Hunt, CEO of Alex TLC, a charity she
set up for patients with adrenoleukodystrophy set up for patients with adrenoleukodystrophy carrier and her two sons had tested positive for the condition. ALD is an X-linked neurological
ADRENOLEUKODYSTROPHY

disorder that destroys myelin, the protective sheath surrounding neurons. Hunt compellingly provides evidence for the introduction of ALD into NBS programmes; sadly, her eldest son, Alex, was diagnosed too late. After he was finally given a neurological test he was told he had between 12 and 18 months to live, and died aged 19 years old. Prior to diagnosis, as with Hunt's younger son Aidan, the disease is treatable by bone marrow transplant and he is now a "healthy, transplant and he is now a "healthy, . mpacts of the disease upon herself and the family, as well as Aidan's "threat of further health issues later in life and the repercussions of aggressive chemotherapy." Despite the evidence showing that early intervention is key to treating the disease, the British National Screening Committee conceded that ALD would be added to the list of recommendations only and reviewed to be added to the panel as part of the regular update cycle However the future is promising: the has approved the addition of ALD to their screening programme.

With COVID-19 heightening the global sensitivity to the fragility of health, Hunt implores us to take advantage of this new perspective. The world is ide the answers, and therefore now is the time to push healthcare systems to prioritise screening of these conditions so that prevention is possible and patients can be informed and empowered to take control of their long-term health.

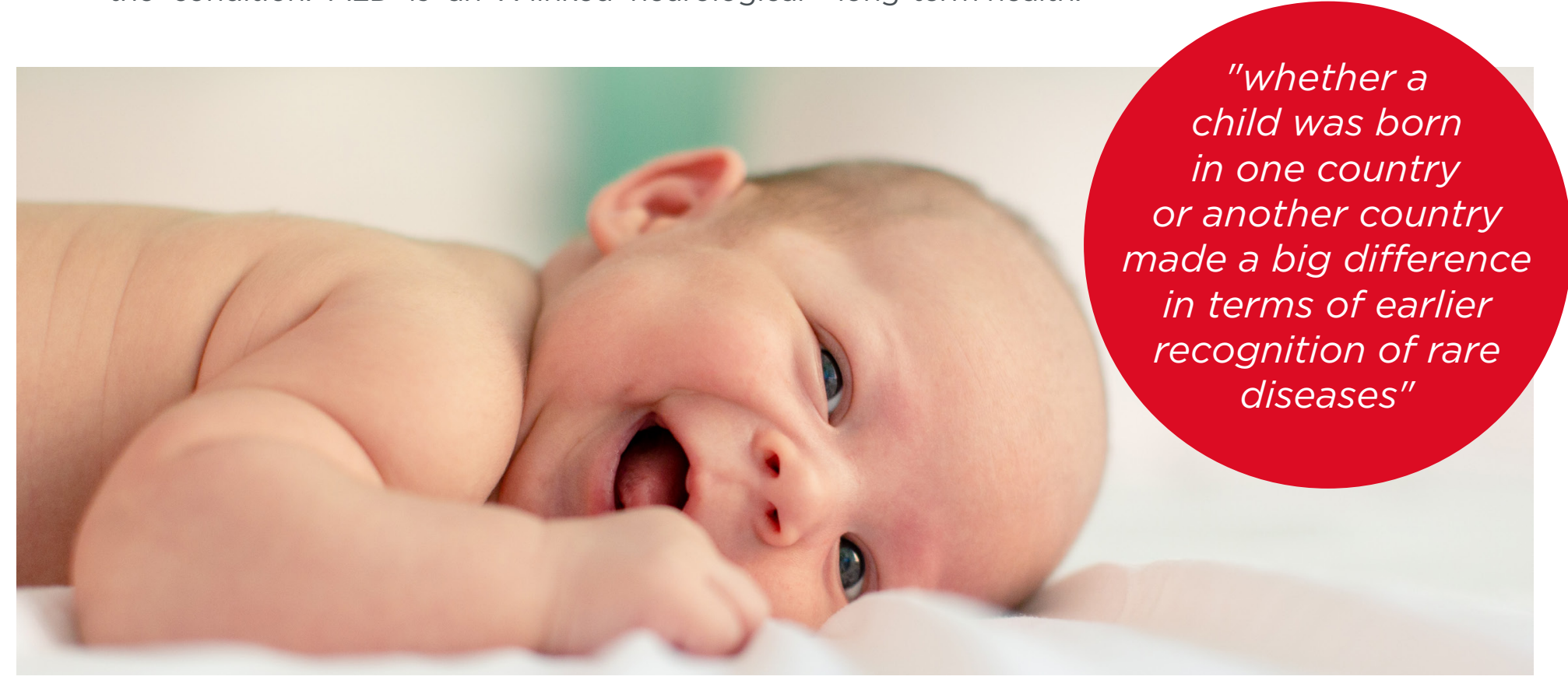

